\title{
ACTUAL PLAYS : QUAND LE JEU DE RÔLE SUR TABLE SE MÉDIATISE
}

\author{
Ugo Roux ${ }^{1}$
}

\begin{abstract}
On observe depuis quelques années la diffusion d'émissions qui présentent des parties de jeu de rôle sur table : les actual plays. Les actual plays répondent à des enjeux médiatiques qui se manifestent en termes d'auditoire, de notoriété et de divertissement. Des moyens conséquents sont alors mobilisés pour développer ces émissions afin de les rendre les plus attractives possibles pour les spectateurs comme pour les joueurs. Il apparaît cependant qu'aucune étude n'ait jamais formalisé ces moyens. L'objectif du présent travail est donc de faire ressortir les modalités de médiatisation et de spectatorisation des actual plays afin de les examiner et de voir si celles-ci font appel à une standardisation ou une fragmentation des pratiques. Pour ce faire, nous avons réalisé une analyse comparative exploratoire sur la base d'une grille d'analyse créée ad hoc. La comparaison semble dégager un modèle de pratiques standardisées dans les actual plays.
\end{abstract}

Le jeu de rôle sur table (aussi appelé indifféremment « jeu de rôle papier » ou « JdR ») est un format de jeu de société et de simulation où les joueurs (rôlistes) développent coopérativement une fiction ludique à travers des personnages qu'ils interprètent dans un univers fictionnel. Ces vecteurs et dispositifs immersifs (Caïra [2007] parle de

1 Ugo Roux est docteur en sciences de l'information et de la communication à l'Institut méditerranéen des Sciences de l'Information et de la Communication (IMSIC) - EA 7492.

Recherches en communication, $n^{\circ} 49$ - Article publié le 14/07/2020 
« véhicule ») que sont les personnages permettent aux joueurs d'interagir virtuellement avec l'univers de jeu comme ils le désirent dans les limites édictées par le besoin de cohérence de celui-ci (Fine, 2002). Un minimum de deux joueurs est nécessaire pour créer le cadre du jeu par un échange verbalisé (généralement oralisé) ; c'est la condition indispensable à l'actualisation du contexte imaginaire (Shulga, 2003) et au développement de la fiction ludique à partir d'une situation initiale. L'un de ces joueurs qui est traditionnellement le maître du jeu - aussi désigné « MJ », « meneur de jeu », ou encore, selon les terminologies propres aux divers jeux de rôle sur table, « maître du donjon » (Donjons \& Dragons [Dungeons \& Dragons en anglais]), " gardien des arcanes » (l'Appel de Cthulhu [The Call of Cthulhu en anglais]), etc. - assure la médiation pragmatique entre les autres joueurs et l'univers de jeu ; il est celui qui a habituellement la responsabilité de gérer tout l'univers de jeu extérieur aux personnages des joueurs ainsi que sa cohérence.

Le jeu de rôle sur table voit fréquemment sa pratique, sa consommation et sa monstration évoluer avec l'introduction des technologies de l'information et de la communication (Roux, 2016 ; Roux \& Roques, 2018 ; Roux et al., 2018). Une activité sociale et médiatique en particulier, le streaming ${ }^{2}$, a fait naître de nouveaux usages et comportements chez certains rôlistes et amène à repenser et redéfinir les logiques $\mathrm{du}$ jeu, notamment en ce qui concerne ses frontières spatiales et temporelles ainsi que ses plans de présence et ses cadres de participation ${ }^{3}$ (Roux \& Roques, 2018 ; Roux et al., 2018).

Le streaming a connu son essor avec le développement des « télévisions sociales » (Cesar \& Geerts, 2011) comme YouTube, Dailymotion et plus récemment Twitch et Mixer pour ne citer qu'elles. Ces dispositifs socionumériques mettent les usagers au centre de leur fonctionnement en sollicitant leur participation qui en est le moteur (Burgess \& Green, 2009). Ces plateformes leur permettent notamment d'y héberger, de diffuser et de partager des contenus vidéo créés par eux-mêmes,

2 Le streaming peut être entendu comme solution technique ou comme activité culturelle (Sjöblom \& Hamari, 2017).

3 Il existe plusieurs cadres de participation - qui vont de l'espace commun de communication au contexte imaginaire de jeu - qui s'emboîtent dans le jeu de rôle sur table (Fine, 2002 ; Shulga, 2003). Ils produisent « un espace combiné de présence dans lequel l'interaction des joueurs se déroule et à l'intérieur duquel ils passent constamment d'un plan de présence à un autre » (Shulga, 2003, p. 106); à savoir, l'action de jeu intradiégétique, l'action ludique métafictionnelle et le cadre réel extradiégétique et extraludique (nous développons plus loin ces notions). 
de bâtir un auditoire, de communiquer et d'interagir avec celui-ci - ces possibilités sont d'autant plus grandes avec les fonctionnalités interconnectées avec d'autres médias sociaux. La production audiovisuelle connaît dès lors une démocratisation et n'est plus l'apanage des grandes entreprises, des grandes institutions et des grands médias (Sjöblom \& Hamari, 2017 ; Sjöblom et al., 2017, 2019). On observe même parfois une professionnalisation de cette activité via des modèles économiques de donation et de monétisation des contenus qui assurent une rémunération des créateurs.

L'apparition de ces services a donc transformé nos comportements en matière de consommation et de production audiovisuelle. On peut notamment le constater avec la diminution de la consommation de contenus télévisuels et l'augmentation de la consommation de contenus sur des services de télévision numérique comme YouTube (Sjöblom et al., 2019). Parmi ces derniers contenus, une catégorie en particulier semble se démarquer par sa popularité au vu des centaines de millions de vues qu'elle génère : le jeu (avec le jeu vidéo en porte-étendard).

Dans ce contexte, on observe depuis quelques années, dans des proportions toutefois bien moindres, la diffusion d'émissions qui présentent des parties de jeu de rôle sur table. Ces émissions - qui sont appelées actual plays - peuvent être diffusées en direct (live streaming en anglais) ou être enregistrées sur des plateformes comme Twitch ou YouTube. Parmi les actual plays les plus connus, nous pouvons notamment citer Rôle'n Play, Donjons \& Jambons et Aventures en France, Critical Role aux États-Unis, Dans le Donjon d'Es-Tu Game au Québec/Canada, ou encore Yogsquest au Royaume-Uni. Le plus vieil enregistrement de ce type d'émissions semble remonter au 5 septembre 2010 avec le programme PAX Prime 2010 D\&D Game d'Acquisitions Incorporated $^{4}$ (Dungeons \& Dragons, 2010).

Avec ces émissions, Roux et Roques (2018) ont fait remarquer que le jeu de rôle sur table devenait un « semi-loisir » (Dumazedier, 1974). « Le semi-loisir est une activité mixte où le loisir est mêlé à une obligation institutionnelle » (Dumazedier, 1974, p. 97). Effectivement, les actual plays répondent à des enjeux médiatiques qui se manifestent en termes d'auditoire, de notoriété et de divertissement. Des moyens conséquents sont ainsi mobilisés pour développer ces émissions afin

4 Voir notamment le site d'Acquisitions Incorporated (http://www.acq-inc.com/portfolio) ainsi que la vidéo « PAX Prime 2010 D\&D Game » (https://youtu.be/uqXqK3ZlqWI). 
de les rendre les plus attractives possible pour les spectateurs comme pour les joueurs. "En effet, entre génériques et réalisation soignés, costumes travaillés, angles de caméras optimisés et autres moyens mobilisés (p. ex. studios d'enregistrement pleinement équipés, équipes techniques), ces émissions adoptent les codes de certains formats télévisuels. » (Roux \& Roques, 2018).

Il apparaît cependant qu'aucune étude n'ait jamais formalisé ces moyens. L'objectif du présent travail est donc de faire ressortir les modalités de médiatisation et de spectatorisation des actual plays afin de les examiner et de voir si celles-ci font appel à une standardisation ou une fragmentation des pratiques. Comme sur ce point les approches tant théoriques qu'empiriques semblent limitées, nous avons réalisé une analyse comparative exploratoire sur la base d'une grille d'analyse créée ad hoc. La comparaison semble dégager un modèle sous-jacent de pratiques standardisées dans les actual plays « à succès ».

\section{Matériel et méthodes}

Dans le cadre de cette étude, nous nous sommes intéressés aux actual plays « à succès »; c'est-à-dire ceux qui se démarquent par leur notoriété. Cette notoriété permet de garantir une certaine représentativité des pratiques ainsi observées. Pour constituer un tel corpus nous avons décidé de restreindre notre sélection aux actual plays qui remplissent les critères suivants :

- l'émission est en français - pour des raisons de compréhension;

- le matériel est publiquement accessible sur les plateformes YouTube, Twitch et/ou Dailymotion - même si certains contenus sont parfois uniquement accessibles aux abonnés ;

- les épisodes atteignent régulièrement plusieurs dizaines de milliers de vues - pour coller, toute proportion gardée en considérant la niche que peut représenter le jeu de rôle sur table, aux exigences énoncées de succès médiatique.

S'agissant d'une étude exploratoire, nous avons retenu trois actual plays qui répondent à ces exigences ; à savoir, Rôle'n Play (Rôle'n Play), Aventures (Bazar du Grenier) et Donjons \& Jambons (OgamingTV et Multigaming Replays FR). Le tableau 1 propose un court comparatif des éléments de diffusion de ces émissions. 
Tableau 1. Comparatif des éléments de diffusion des actual plays Rôle'n Play, Aventures et Donjons \& Jambons

\begin{tabular}{lccc} 
& $\begin{array}{c}\text { Rôle'n Play } \\
\text { (Rôle'n Play) }\end{array}$ & $\begin{array}{c}\text { Aventures } \\
\text { (Bazar du Grenier) }\end{array}$ & $\begin{array}{c}\text { Donjons \& Jambons } \\
\text { (OgamingTV \& } \\
\text { Multigaming } \\
\text { Replays FR) }\end{array}$ \\
\hline $\begin{array}{l}\text { Plateforme de diffusion } \\
\text { principale }\end{array}$ & YouTube & YouTube (VOD) & Twitch (direct) \\
\hline $\begin{array}{l}\text { Plateforme de diffusion } \\
\text { secondaire }\end{array}$ & Dailymotion, Twitch & YouTube (VOD) \\
\hline $\begin{array}{l}\text { Mode de diffusion } \\
\text { principal }\end{array}$ & VOD (montée) & VOD (montée) & Direct > VOD \\
\hline $\begin{array}{l}\text { Mode de diffusion } \\
\text { secondaire }\end{array}$ & Direct $>$ VOD & Direct $>$ VOD & VOD \\
\hline Planning de diffusion & $\checkmark$ & $\checkmark$ & $\checkmark$ \\
\hline $\begin{array}{l}\text { Activité principale de la } \\
\text { chaîne }\end{array}$ & $\checkmark$ & & \\
\hline Chaîne dédiée & $\checkmark$ & & \\
\hline
\end{tabular}

Nous avons visionné l'intégralité de la production de ces trois émissions (p. ex. épisodes, hors-séries, foires aux questions [FAQ], teasers). Pour les comparer, nous avons créé une grille d'analyse ad hoc en nous inspirant des travaux de Sjöblom et al. (2019). Ces auteurs ont en effet développé une grille d'analyse afin de comparer les pratiques médiatiques des streameurs ${ }^{5}$ les plus populaires sur Twitch, et ce, afin d'en dégager les éléments récurrents. Afin de correspondre à notre objet de recherche, cette grille de départ a évolué au fur et à mesure de nos observations des méthodes de réalisation perçues ou déclarées.

\section{2. «Spectatoriser » le jeu de rôle sur table}

Le jeu de rôle sur table est une activité traditionnellement discrète et faiblement tournée vers l'extérieur (Beau, 2017) « avec une présence médiatique qui se structurait jusqu'à récemment principalement autour d'une presse, de podcasts, de forums et autres sites Internet tous spécialisés » (Roux \& Roques, 2018).

5 Créateurs de contenus qui pratiquent le streaming. 
Le jeu de rôle est peu connu cependant. Il est tourné vers l'intérieur, pratiqué par de petits groupes dans l'intimité du domicile privé. Il échappe à la connaissance informelle que tout acteur social peut avoir des activités des « autres » (qu'est-ce que les gens font ?), faite d'informations directes et personnelles, d'expériences communes, de simples regards et de médiatisation. Cette faible visibilité tient sans doute aussi à son mode de diffusion : loin du prosélytisme, il s'insinue par « initiation »- mot consacré des joueurs pour désigner leur premier apprentissage du jeu - et sans être secrète, c'est une pratique peu bavarde sur elle-même, dont les joueurs ne font pas ostentation. (Voelckel, 1995, p. 58).

Pourtant, comme nous l'avons dit, avec l'apparition et le développement des actual plays, le jeu de rôle papier sort de plus en plus de son « petit $[\ldots]$ monde social » (Fine, 2002, p. 1) pour se tourner vers un auditoire distant dans l'espace et parfois le temps, et ce, grâce aux plateformes de streaming. Cette remédiation entraîne son lot de mutations de la pratique du jeu de rôle sur table, de sa monstration et de sa consommation. En effet, intégrer volontairement des spectateurs dans le jeu comme troisième partie prenante nécessite de créer et de maintenir de nouvelles formes d'engagement vis-à-vis d'eux pour compenser le fait qu'ils ne sont pas acteurs dans la diégèse et sont plongés dans la même « pauvreté sensorielle » que les joueurs et le maître du jeu (Caïra, 2007). D'autant plus que la consommation d'actual plays constitue une forme non intuitive de consommation de médias puisque la satisfaction retirée des jeux est normalement due à l'interaction directe avec ceux-ci (Roux \& Roques, 2018). " L'intégration du spectateur devient dès lors un des enjeux et l'une des exigences de divertissement du "contrat" qui lie les rôlistes sur la bonne manière de conduire la partie (Caïra, 2007). » (Roux \& Roques, 2018). Il ne s'agit désormais plus seulement de développer l'expérience de jeu mais également l'expérience spectatorielle.

Dans cette partie, nous mettrons en évidence deux voies majeures par lesquelles passent la médiatisation du jeu de rôle sur table et par conséquent l'intégration du spectateur au jeu ; à savoir, le degré de préparation d'une partie de jeu de rôle d'une part, et l'interaction avec les spectateurs d'autre part. 


\subsection{Sophistiquer la préparation}

Le premier et le plus évident moyen pour faire rentrer les joueurs et les spectateurs dans le « cercle magique ${ }^{6}$ » passe par la mise en scène de la partie de jeu de rôle sur table - cette tâche revient traditionnellement au maître du jeu. Il est d'autant plus compliqué de créer ce " cercle magique » que le jeu de rôle papier a un caractère fortement dématérialisé et qu'il se définit par « une activité essentiellement cognitive et l'imaginaire des joueurs en est le "terrain de jeu" » (Roux, 2016). Le maître du jeu doit ainsi créer et maintenir la médiation pragmatique entre les joueurs et l'univers de jeu - qu'il crée, reprend ou adapte dont il doit communiquer les propriétés par le « contrat fictionnel ${ }^{7}$ » (Tychsen et al., 2005, p. 216). Pour l'aider dans cette tâche, le maître du jeu fait fréquemment attention à la préparation d'une partie - par exemple en préparant avec soin le scénario, les aides de jeu, le décor, l'ambiance ou encore les costumes ; l'objectif est d'améliorer notamment l'évocation et le rythme dont la finalité commune est l'immersion dans l'univers ludo-fictionnel.

La sophistication de la préparation peut donc se traduire par le soin apporté à tout ce qui peut contribuer à l'expérience de jeu - et à l'expérience spectatorielle pour les actual plays. Dans ce dernier cas, la production de ceux-ci est parfois tellement performante que le maître du jeu va jusqu'à mobiliser des moyens humains et techniques considérables pour rendre l'émission attractive (p. ex. matériel audiovisuel, équipe technique, studio, effets visuels et spéciaux). Un exemple particulièrement parlant de cela est celui de Rôle'n Play qui compte 28 personnes réparties sur 13 postes différents de production (visibles dans les crédits de fin de chaque épisode). Le tableau 2 présente une synthèse des éléments de préparation relevés dans les actual plays comparés.

6 La notion de « cercle magique » (Salen \& Zimmerman, 2004) renvoie à la frontière entre le monde réel et le monde fantasmé du jeu; lorsque l'on rentre dans le « cercle magique », on « quitte » le monde réel pour rentrer dans le monde fictionnel. Cette notion est empruntée à Huizinga (1938).

7 La notion de contrat fictionnel renvoie à l'accord entre les joueurs sur le fonctionnement du monde fictionnel et sur les règles qui le régissent. 
Tableau 2. Analyse comparative des éléments de préparation des actual plays Rôle'n Play, Aventures et Donjons \& Jambons

\begin{tabular}{|c|c|c|c|}
\hline & $\begin{array}{l}\text { Rôle'n Play } \\
\text { (Rôle'n Play) }\end{array}$ & $\begin{array}{c}\text { Aventures } \\
\text { (Bazar du Gre- } \\
\text { nier) }\end{array}$ & $\begin{array}{l}\text { Donjons \& Jambons } \\
\text { (OgamingTV \& Mul- } \\
\text { tigaming Replays FR) }\end{array}$ \\
\hline \multicolumn{4}{|c|}{ Préparation } \\
\hline Mode de jeu & Présentiel & Distanciel & Présentiel \\
\hline Post-production & $\checkmark$ & $\checkmark$ & \\
\hline Génériques & $\checkmark$ & $\checkmark$ & $\checkmark$ \\
\hline - Générique de début & $\checkmark$ & $\checkmark$ & $\checkmark$ \\
\hline - Générique de fin & $\checkmark$ & $\checkmark$ & \\
\hline $\begin{array}{l}\text { - Générique de présen- } \\
\text { tation des joueurs/ } \\
\text { personnages }\end{array}$ & $\checkmark$ & $\checkmark$ & $\checkmark$ \\
\hline Studio & $\checkmark$ & & $\checkmark$ \\
\hline Équipe technique & $\checkmark$ & $\checkmark$ & $\checkmark$ \\
\hline Décor & $\checkmark$ & & $\checkmark$ \\
\hline - Décor évocateur & & & $\checkmark$ \\
\hline Costumes & $\checkmark$ & & $\checkmark$ \\
\hline $\begin{array}{l}\text { Ambiance sonore } \\
\text { et musicale }\end{array}$ & $\checkmark$ & $\checkmark$ & $\checkmark$ \\
\hline Effets spéciaux & $\checkmark$ & & $\checkmark$ \\
\hline $\begin{array}{l}\text { Angles de caméras } \\
\text { multiples }\end{array}$ & $\checkmark$ & & $\checkmark$ \\
\hline Overlays & $\checkmark$ & $\checkmark$ & $\checkmark$ \\
\hline Incrustation d'illustrations & $\checkmark$ & $\checkmark$ & $\checkmark$ \\
\hline $\begin{array}{l}\text { Résumés des épisodes } \\
\text { précédents }\end{array}$ & $\checkmark$ & $\checkmark$ & $\checkmark$ \\
\hline $\begin{array}{l}\text { Explication du JdR et du } \\
\text { système de règles }\end{array}$ & $\checkmark$ & $\checkmark$ & $\checkmark$ \\
\hline $\begin{array}{l}\text { Simplification du système } \\
\text { de règles }\end{array}$ & & $\checkmark$ & $\checkmark$ \\
\hline $\begin{array}{l}\text { Invités (célébrités, specta- } \\
\text { teurs, etc.) }\end{array}$ & $\checkmark$ & $\checkmark$ & $\checkmark$ \\
\hline Voix off (résumés, etc.) & & & $\checkmark$ \\
\hline Vocable de séries & $\checkmark$ & $\checkmark$ & $\checkmark$ \\
\hline - Cycle & & & $\checkmark$ \\
\hline - Arc & & $\checkmark$ & \\
\hline
\end{tabular}


ACTUAL PLAYS : QUAND LE JEU DE RÔLE SE MÉDIATISE 141

\begin{tabular}{|c|c|c|c|}
\hline - Saison & $\checkmark$ & $\checkmark$ & $\checkmark$ \\
\hline - Épisode & $\checkmark$ & $\checkmark$ & $\checkmark$ \\
\hline Différents genres de jeu & $\checkmark$ & $\checkmark$ & $\checkmark$ \\
\hline Différents MJ & $\checkmark$ & $\checkmark$ & $\checkmark$ \\
\hline Différents joueurs & $\checkmark$ & $\checkmark$ & $\checkmark$ \\
\hline Comédiens & $\checkmark$ & & \\
\hline Roleplay & $\checkmark$ & $\checkmark$ & $\checkmark$ \\
\hline $\begin{array}{l}\text { Épisodes hors-séries, } \\
\text { spin-off, etc. }\end{array}$ & $\checkmark$ & $\checkmark$ & $\checkmark$ \\
\hline $\begin{array}{l}\text { Épisode } 0, \text { épisode de } \\
\text { mi-saison, etc. }\end{array}$ & $\checkmark$ & $\checkmark$ & \\
\hline Épisode pilote, prototype & $?$ & $\checkmark$ & $?$ \\
\hline $\begin{array}{l}\text { Teasers, vidéos annonces, } \\
\text { etc. }\end{array}$ & $\checkmark$ & $\checkmark$ & $\checkmark$ \\
\hline $\begin{array}{l}\text { Débriefing fin d'épisode, } \\
\text { saison, etc. }\end{array}$ & $\checkmark$ & $\checkmark$ & $\checkmark$ \\
\hline Réunions, débriefings en off & $\checkmark$ & $\checkmark$ & $\checkmark$ \\
\hline Partenariats & $\checkmark$ & & \\
\hline Financement participatif & $\checkmark$ & $\checkmark$ & \\
\hline
\end{tabular}

La mise en scène passe prioritairement par le sensible. Le jeu de rôle papier est, comme nous l'avons dit, une forme particulière de jeu de société qui repose sur la construction collaborative d'une fiction et d'un univers de jeu à travers l'imagination et les interactions des joueurs. Ce caractère essentiellement dématérialisé du jeu de rôle sur table pose le problème de la convergence imaginaire collective des joueurs par rapport au contexte ludique proposé par le maître du jeu. C'est pourquoi le maître du jeu s'appuie fréquemment sur des moyens qui l'aident à donner forme au monde qu'il décrit. Cela peut se traduire par l'usage, entre autres, d'images (p. ex. de paysages, de situations, de personnages ou de créatures) et/ou de sons (ambiance sonore et/ou musicale) (figure 1). Ces usages servent dans un premier temps à illustrer les situations narrées et ainsi à renforcer l'immersion en sollicitant et en stimulant les sens des parties prenantes au jeu, et, dans un second temps qui est la conséquence du précédent, à réduire les dissonances interprétatives et à harmoniser une vision commune de l'univers de jeu.

Dans le cadre d'actual plays, la médiatisation de ces ressources auprès des spectateurs passe préférablement par l'usage de technologies de l'information et de la communication (p. ex. applications, logiciels, smartphones, tablettes, ordinateurs). L'usage de dispositifs numé- 
riques est particulièrement manifeste dans Aventures où est utilisée la table virtuelle Roll20 qui permet de présenter visuellement la scène, les décors et la position des personnages.

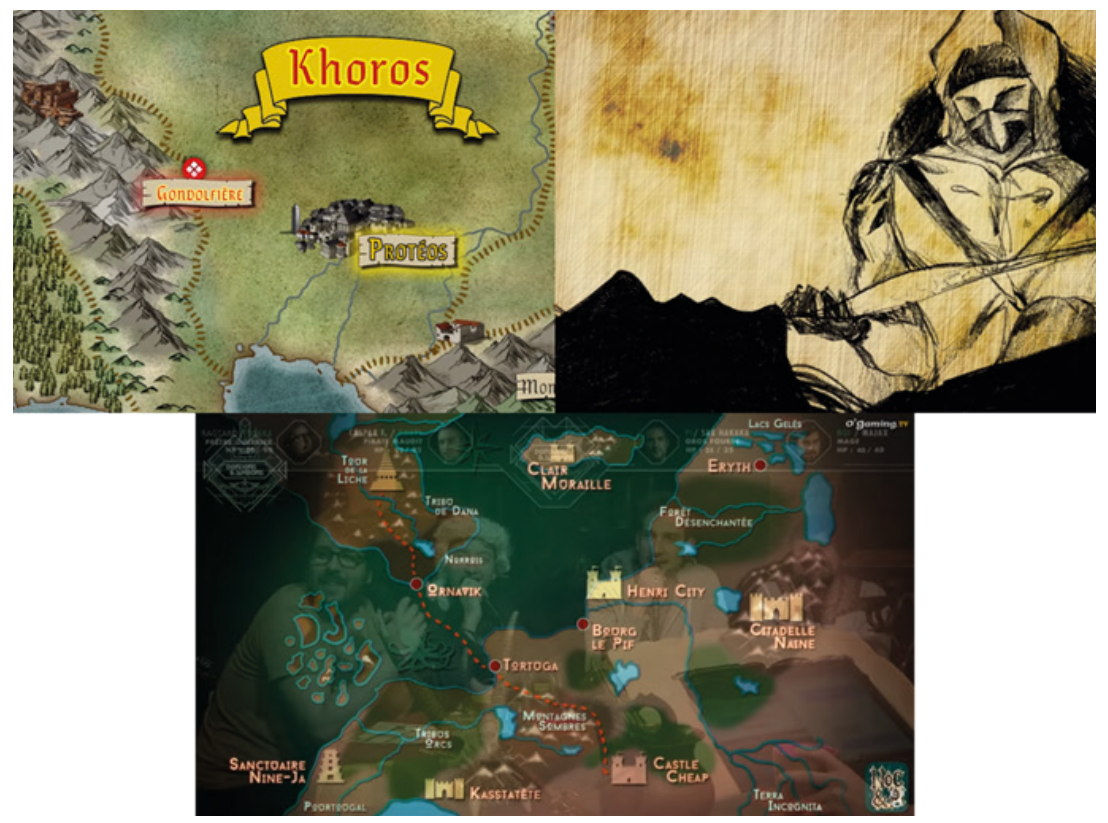

Figure 1. En haut à gauche, une carte dans Rôle'n Play (source : https://youtu.be/zw317XgHur4); en haut à droite, une illustration d'une situation dans Aventures (source : https://youtu.be/ZJ_uZSurIBM) ; en bas, une carte dans Donjons \& Jambons (source : https://youtu.be/0e_y-Fszh1Y)

La mise en scène passe également par la posture des joueurs. Comme il a été dit précédemment, le jeu de rôle sur table permet au joueur « de s'actualiser dans le cadre du jeu comme s'il n'était pas luimême mais un autre être vivant dans un autre environnement » (Shulga, 2003, p. 103), et ce, grâce à son personnage qu'il interprète. Cette interprétation peut connaître des degrés de projection identitaire variables (Roux, s. p.). On observe dans les actual plays que les joueurs adoptent dans la plupart des cas une posture roleplay qui s'inscrit dans le plan le plus immersif de l'action de jeu où se produisent les événements intradiégétiques. Roux (2016) dit du roleplay qu'il « correspond à l'interprétation immersive et créative que fait un joueur de son personnage ». De plus, il faut comprendre que « cette interprétation doit non seulement se faire dans un souci de réalisme et de cohérence intradiégétique, mais 
également dans un but de renforcement et d'enrichissement de la réalité fictionnelle dans laquelle elle s'inscrit ». Dans un roleplay qui se veut encore plus poussé, on observe parfois que les joueurs sont costumés ; ce qui est fréquemment le cas dans Donjons \& Jambons (figure 2). Il est intéressant de voir, à propos de cette notion de roleplay, la vidéo FAQ réalisée par l'équipe de Rôle'n Play ${ }^{8}$. En effet, les joueurs étant des comédiens de profession, ils y développent de manière très riche et détaillée leur façon de jouer leur personnage.

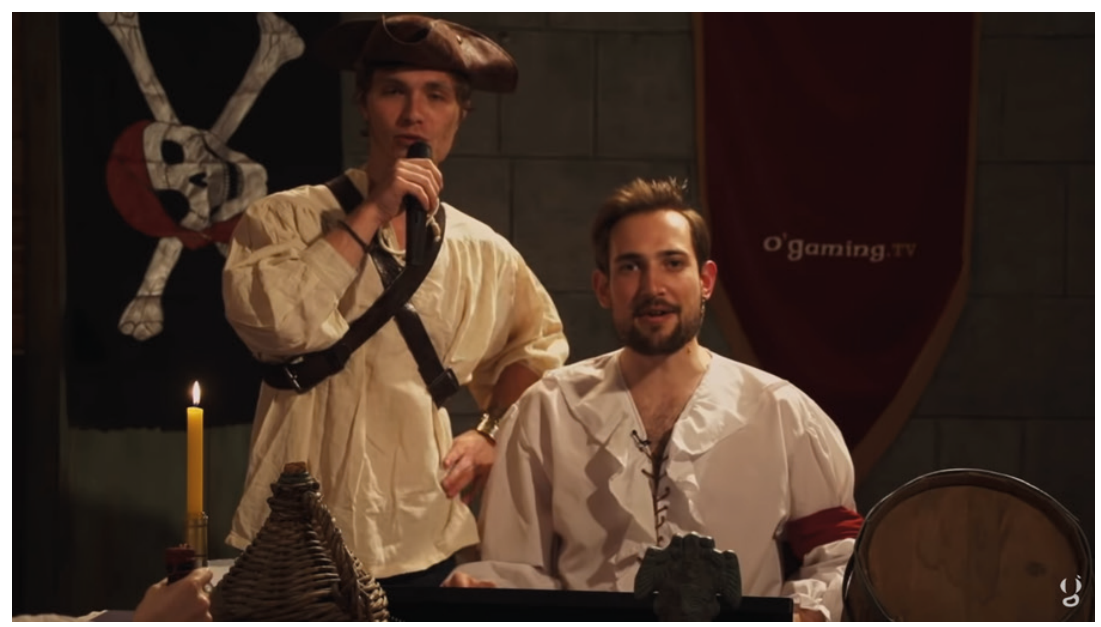

Figure 2. Des joueurs costumés dans Donjons \& Jambons (source : https://youtu.be/o9GDCvgBPeI)

Conséquemment au précédent point, on observe aussi une normalisation de l'attitude des joueurs. Comme nous avons pu le voir, l'univers de jeu et la fiction se construisent à mesure que les interactions entre joueurs se développent. Cette contribution plurielle à cette création se fait en respectant une convention d'intervention plus ou moins définie que Dauphragne (2008) qualifie de " récit à plusieurs voix ». Cela peut se traduire, entre autres, par un ordre de prise de parole, des temps de parole équivalents ou des auto-censures. Aventures constitue un bon exemple de ces prises de parole normalisées puisque celles-ci sont définies selon un ordre précis d'intervention des joueurs.

Malgré cette normalisation de la prise de parole qui doit être profitable à l'expérience de jeu, il arrive souvent que des interventions

8 Source : https://youtu.be/7p-Yrq5fY3g 
contreviennent à celle-ci. Par exemple, il est fréquent d'observer des moments parasites, comme les dialogues de commentaires, qui peuvent nuire par leur excès à une partie de jeu de rôle papier en ralentissant le rythme (Fine, 2002 ; Caïra, 2007). Pour éviter ces moments de forte digression qui pourraient alourdir le montage en post-production d'une part, et se révéler perturbateurs pour le spectateur en faisant chuter son attention et son intérêt d'autre part, les rôlistes peuvent réguler leurs interventions sinon les retirer en dernier recours en post-production. C'est ainsi que nous avons pu remarquer plusieurs fois, dans les émissions étudiées, des coupes dans le montage qui laissent deviner la suppression de séquences inutiles. Julien Dutel - le maître du jeu de Rôle’n Play - précise à ce propos qu'il souhaite tourner « dans les conditions du live » afin, justement, de ne pas casser le rythme et de ne pas alourdir le montage en post-production; dans ces conditions, seulement une à deux minutes sont finalement retirées par épisode au moment du montage9 ${ }^{9}$.

\subsection{Interagir avec les spectateurs}

Le second moyen d'engagement du spectateur passe aussi par la mise en interaction de la partie de jeu de rôle sur table. Pour ce faire, les créateurs de contenus ont recours aux « télévisions sociales » (Cesar $\&$ Geerts, 2011) dont le principal moteur est, comme pour tout média social, la culture participative (Burgess \& Green, 2009). Parmi ces dispositifs, le streaming en particulier propose un canal communicationnel très complet avec un haut niveau d'interaction (Sjöblom \& Hamari, 2017) qui permet une « coprésence écranique ${ }^{10}$ » (Beaudouin, 2002). Il permet ainsi d'étendre à un auditoire distant dans l'espace et parfois le temps ${ }^{11}$ les cadres de participation ainsi que les plans de présence du jeu de rôle papier (Roux \& Roques, 2018 ; Roux et al., 2018) - ce qui est particulièrement vrai dans le cas du live streaming qui permet d'interagir en synchronie avec les créateurs de contenus malgré la distance. Le tableau 3 présente une synthèse des différents éléments de préparation relevés dans les actual plays comparés.

9 Source : https://youtu.be/7p-Yrq5fY3g.

10 Cette forme de coprésence consiste à « partager un même contexte, une même situation définie par un ensemble de textes disponibles à l'écran » (Beaudouin, 2002, p. 219).

11 «La médiation de l'informatique connectée déspatialise et désynchronise l'acte de monstration et le moment de sa réception » (Granjon, 2012, p. 126). 
Tableau 3. Analyse comparative des éléments d'interaction des actual plays Rôle'n Play, Aventures et Donjons \& Jambons

\begin{tabular}{lccc} 
& $\begin{array}{c}\text { Rôle'n Play } \\
\text { (Rôle'n Play) }\end{array}$ & $\begin{array}{c}\text { Aventures } \\
\text { (Bazar du grenier) }\end{array}$ & $\begin{array}{c}\text { Donjons \& Jambons } \\
\text { (OgamingTV } \\
\text { \& Multigaming } \\
\text { Replays FR) }\end{array}$ \\
\hline Activités spectactorielles & $\checkmark$ & $\checkmark$ & $\checkmark$ \\
\hline Commentaire & $\checkmark$ & $\checkmark$ & $\checkmark$ \\
\hline Contribution & $\checkmark$ & $\checkmark$ & $\checkmark$ \\
\hline Création & $\checkmark$ & $\checkmark$ & $\checkmark$ \\
\hline $\begin{array}{l}\text { S'adresser aux specta- } \\
\text { teurs face caméra }\end{array}$ & $\checkmark$ & $\checkmark$ & $\checkmark$ \\
\hline Médias sociaux officiels & $\checkmark$ & $\checkmark$ & $\checkmark$ \\
\hline Sollicitation feedbacks & $\checkmark$ & $\checkmark$ & $\checkmark$ \\
\hline FAQ & $\checkmark$ & $\checkmark$ & $\checkmark$ \\
\hline Rencontres communauté & $\checkmark$ & $\checkmark$ & $\checkmark$ \\
\hline Modérateurs & $\checkmark$ & $\checkmark$ & $\checkmark$ \\
\hline Publication des lores & & & $\checkmark$ \\
\hline
\end{tabular}

Pour rendre compte de ce passage d'une posture passive à une posture active et participative, Roux et Roques (2018) ont mobilisé la notion d'activités « spectactorielles ${ }^{12}$ » pour désigner la nature de ces nouvelles interactions entre les spectateurs et le jeu. Les dispositifs « transmédias » permettent justement cette inversion des positions des spectateurs pour les transformer en « spectacteurs » (Weissberg, 2009), l'accent étant mis sur les possibilités d'interaction de l'individu plutôt que sur sa position de protagoniste. On peut ainsi dégager trois types d'interactions spectactorielles dans les actual plays sur la base des travaux de Rebillard (2007), Schmitt (2015) et Roux et Roques (2018) : le commentaire, la création et la contribution. "Sans être joueur le spectateur devient tout de même un des participants au jeu; sa posture est plus passive que celle $\mathrm{du}$ joueur mais elle est toutefois plus active que celle du simple spectateur (Sjöblom \& Hamari, 2017). » (Roux \& Roques, 2018).

12 De la notion de « spectacteur » développée par Dumouchel qui dit de celle-ci qu'elle « est un mot-valise contractant le mot spectateur et le mot acteur. Le spectacteur est un spectateur-acteur de l'œuvre interactive. Il est à la fois l'observateur du récit, celui pour qui le récit est dit ou montré, et le protagoniste du récit, celui par qui le récit se joue. » (Dumouchel, 1991, p. 41). 
Les premières formes d'interaction spectateur/jeu se manifestent le plus fréquemment par des activités de commentaire. Les plateformes de streaming mettent généralement à la disposition de leurs usagers des espaces pour commenter de façon asynchrone (espaces commentaires lors des émissions diffusées en différé) ou quasi synchrone (tchats $I R C$ lors des émissions diffusées en direct) ; la figure 3 présente différentes situations d'interactions entre les spectateurs et les créateurs de contenus. Cette prise de parole peut tout à fait être en lien avec le jeu (niveau extradiégétique ${ }^{13}$ et métafictionne ${ }^{14}$ sans incidence sur l'action ludique) ou ne pas du tout l'être (niveau extraludique ${ }^{15}$ dans le cadre réel). Notons que le recours à des modérateurs dédiés est parfois nécessaire pour animer et normaliser ces prises de parole qui peuvent souvent s'avérer très nombreuses et qui empêchent fréquemment les rôlistes de remplir cette tâche.

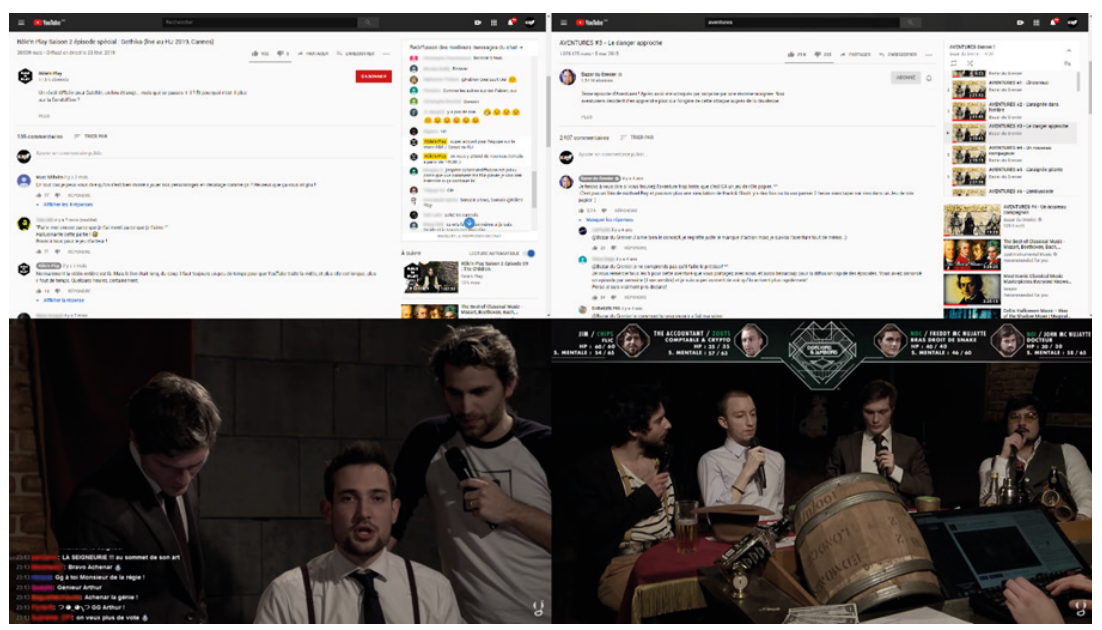

Figure 3. En haut à gauche, l'espace commentaire et le tchat IRC d'un épisode live de Rôle'n Play (source : https://youtu.be/CgU-RscBO3w); en haut à droite, l'espace commentaire d'un épisode d'Aventures (source : https://youtu. be/Dct2gzeoKlc); en bas à gauche, interaction joueurs/spectateurs après un épisode de Donjons \& Jambons (source : https://youtu.be/HrrHJWjmT8s); en bas à droite, le maître du jeu répond au cours d'une partie aux spectateurs de Donjons \& Jambons (source : https://youtu.be/HrrHJWjmT8s)

13 Niveau extérieur à la diégèse ou, autrement dit, extérieur à la fiction et son univers. 14 Niveau d'observation, d'analyse ou encore de commentaire de la fiction.

15 Niveau totalement extérieur au jeu et donc sans rapport avec celui-ci. 
Au-delà des fonctionnalités des dispositifs techniques qui permettent des interactions à distance, une redéfinition des postures des rôlistes s'avère parfois nécessaire pour les normaliser. Dans ce contexte des actual plays, la fonction de maître du jeu est celle dont les missions nous semblent avoir évolué le plus. Effectivement, au-delà de ses traditionnelles attributions, le maître du jeu est désormais fréquemment amené à adopter une posture de « maître de cérémonie 》 auprès d'un public qui n'est plus seulement constitué de ses joueurs (Roux \& Roques, 2018), et ce, afin de provoquer et d'orienter certaines des interactions évoquées précédemment.

Le maître du jeu est en théorie omnipotent dans l'univers ludique où il mastérise, c'est pourquoi on y fait souvent référence en tant que « Dieu ». Ceci signifie en pratique qu'il est le démiurge du monde fictionnel qu'il donne à jouer ; il a l'autorité interprétative, narrative et créative ultime. Il décide, entre autres, des évènements qui s'y produisent, des lois qui le régissent et des créatures qui l'habitent. On observe fréquemment que les interactions entre les spectateurs et le jeu passent justement par un partage de ces prérogatives.

L'interaction spectateur/jeu peut donc passer par la mise à contribution du spectateur en partageant avec lui un peu de l'autorité narrative du maître du jeu. Le jeu de rôle papier est une fiction ludique et, à ce titre, le maître du jeu propose à sa table une trame fictionnelle - qu'il crée, reprend ou adapte - dont il assure la narration principale. Cette trame prend généralement la forme d'un canevas (ou scénario) ${ }^{16} \mathrm{ou}$ d'une campagne plus ou moins élaborée et directive. Le maître du jeu y présente tout d'abord une situation initiale aux joueurs, puis il déroule et adapte son récit de manière dynamique en fonction des actions et réactions de ces derniers. Cette trame est le cœur du jeu de rôle sur table puisque celui-ci ne prend tout son sens que s'il est rapporté à un récit, un contexte imaginaire, un univers de fiction (Dauphragne, 2011a, 2011b).

À l'instar de tout récit, l'intégralité de l'intrigue n'est donc dévoilée qu'à mesure que l'on y progresse. Le maître du jeu a donc pour tâche de distiller les éléments de l'intrigue et par conséquent de maintenir la naïveté de ses joueurs - et de son public - sur la suite des événements.

16 Nous préférons le terme de canevas à celui de scénario qui, popularisé par le cinéma, renvoie à une idée de linéarité et de prescription de la fiction. A contrario, le terme de canevas renvoie à une idée d'incomplétude où les joueurs pourront apporter leur contribution à la fiction (de Canteloube, 2015, 2019). 
Cependant, il arrive parfois, lors des actual plays diffusés en direct, que le maître du jeu partage un peu de son autorité en faisant appel en cours de partie à son public pour orienter la trame narrative - ce faisant, le maître du jeu étend son rôle central de médiateur à l'interaction joueurs/spectateurs. Pour ce faire, le maître du jeu a souvent recours à un sondage en temps réel qui propose diverses possibilités d'issues à une situation donnée ; la proposition qui remporte le vote a alors un impact sur l'action ludique puisqu'elle est appliquée en jeu après la fin du suffrage (figure 4).
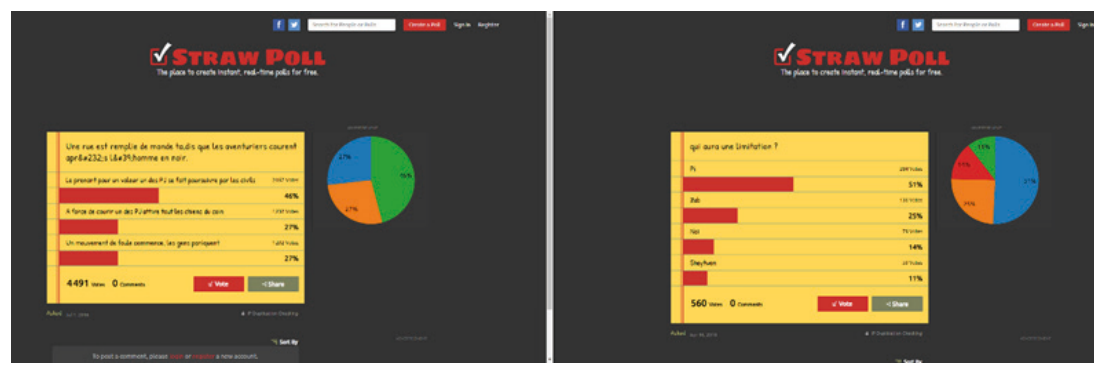

Figure 4. À gauche, un Straw Poll dans Aventures (source Straw Poll : http://www.strawpoll.me/10640598/r ; source vidéo : https://youtu.be/j63Ega_dMaY) ; à droite, un Straw Poll dans Donjons \& Jambons (source Straw Poll : https://www.strawpoll.me/4597621/r ; source vidéo : https://youtu.be/ Ax5OF_SgCPY)

Le maître du jeu peut également mettre à contribution le spectateur en partageant avec lui un peu de son autorité créative. Le jeu de rôle papier propose à jouer des mondes qu'il faut structurer et rendre vivants. Mis à part les personnages joueurs, le maître du jeu a pour responsabilité d'animer le monde par ses créations. Cependant, il arrive parfois que des spectateurs participent à donner forme au monde par leurs propres créations. Cela peut se manifester notamment par la représentation « concrète » d'éléments diégétiques. Par exemple, la figure 5 présente des créations réalisées par des spectateurs; en haut à gauche, des fan arts de personnages dans Rôle'n Play, en haut à droite, des illustrations d'orcs ennemis dans Aventures et, en bas, un amigurumi du personnage « Hamgeon » qui apparaît dans Donjons \& Jambons. Ces deux dernières créations ont d'ailleurs été reprises en jeu pour illustrer des personnages. 


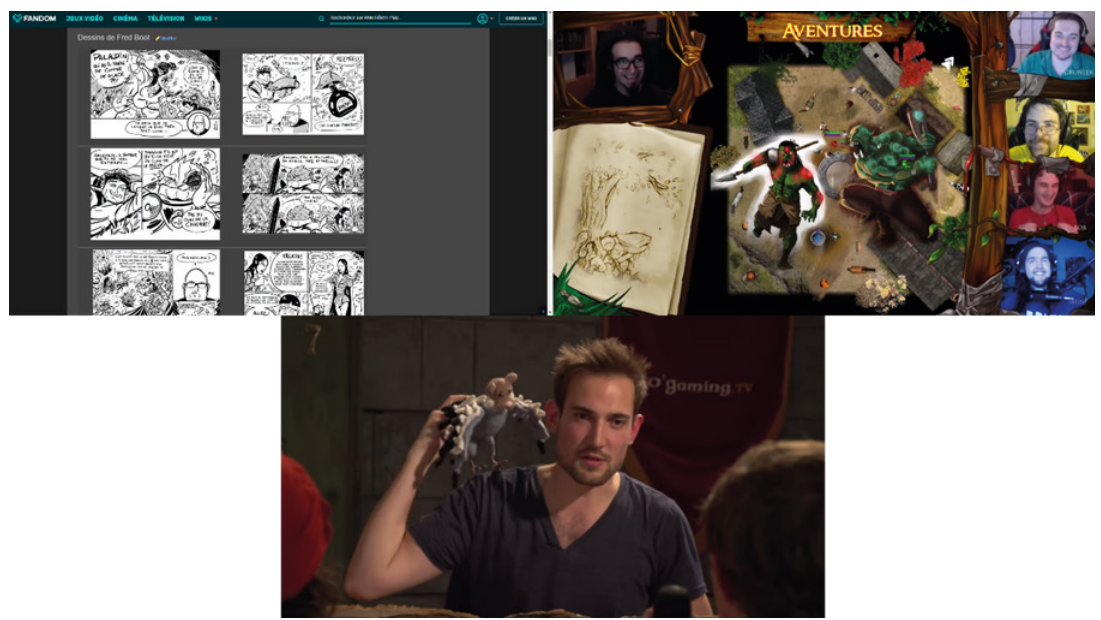

Figure 5. En haut à gauche, des fan arts pour Rôle'n Play (source : https:// role-n-play.fandom.com/fr/wiki/Fan_arts) ; en haut à droite, des illustrations d'orcs dans un épisode d'Aventures (source : https://youtu.be/C2ExaCHx9f8); en bas, un amigurumi du personnage « Hamgeon » dans un épisode de Donjons \& Jambons (source : https://youtu.be/CPrBbU2FLkY)

\section{Conclusion}

Comme nous avons pu le dire précédemment, nous observons depuis quelques années sur les télévisions sociales le développement d'émissions présentant des parties de jeu de rôle papier : les actual plays. Souhaitant répondre à des enjeux médiatiques, ces émissions entrent fréquemment dans une logique de « semi-loisir » (Dumazedier, 1974) et certaines d'entre elles se distinguent par leur niveau élevé de sophistication et de préparation en vue de les rendre plus attractives pour leur auditoire. Des moyens humains et techniques considérables sont ainsi parfois déployés pour répondre à cet objectif (Roux \& Roques, 2018).

Quels sont ces moyens de médiatisation et de spectatorisation ? Répondent-ils à une standardisation ou une fragmentation des pratiques médiatiques des créateurs d'actual plays ? Telles sont les questions auxquelles nous avons tenté de répondre dans cette étude. Pour ce faire, nous avons réalisé une analyse comparative exploratoire de trois actual plays en français « à succès »-Rôle'n Play (Rôle'n Play), Aventures (Bazar du Grenier) et Donjons \& Jambons (OgamingTV et Multigaming Replays FR) - à l'aide d'une grille d'analyse créée ad hoc. 
Cette analyse nous a permis de mettre en évidence deux dimensions importantes d'éléments employés pour médiatiser et spectatoriser les actual plays ; à savoir, la préparation et l'interaction. La préparation renvoie à la production, la réalisation et la matérialisation de la partie de jeu de rôle sur table. On peut par exemple y considérer l'élaboration des costumes, des accessoires et des décors, la postproduction ou encore la mobilisation d'équipes techniques. L'objectif est d'augmenter l'engagement du spectateur en lui proposant un contenu de qualité, tant par son esthétique que son rythme. L'interaction quant à elle renvoie, comme son nom l'indique, aux interactions - ainsi que les moyens déployés pour les susciter - qui s'opèrent entre les actual plays et leurs spectateurs. Celles-ci semblent principalement se décliner sous la forme de commentaires, de contributions et de créations. On parlera alors d'activités spectactorielles puisque l'objectif est de développer l'engagement de l'auditoire en lui faisant adopter une posture plus active que celle du spectateur.

En conclusion, il ressort de notre étude que les actual plays « à succès » semblent reposer sur les mêmes composantes dans leur réalisation ; ce qui laisse présumer l'existence d'un modèle sousjacent pour cette catégorie d'émissions. Le caractère généralisable de ce modèle à l'ensemble des actual plays reste tout de même à vérifier au vu de la nature exploratoire de notre recherche. Dans cette optique, la grille d'analyse ici développée propose une base heuristique intéressante. Effectivement, dans un contexte où les recherches sur le jeu de rôle papier, et encore plus spécifiquement sur les actual plays, demeurent relativement marginales et peu développées, il nous paraît important de déployer des outils et méthodes adaptés.

\section{Références}

Beau, F. (dir.) (2007). Culture d'Univers. Jeux en réseau, mondes virtuels, le nouvel âge de la société numérique, coll. Innovation. Limoges : FYP Éditions.

Beaudouin, V. (2002). De la publication à la conversation : Lecture et écriture électroniques. Réseaux 116, 199-225.

Burgess, J. \& Green, J. (2009). YouTube. Digital Media and Society Series. Cambridge : Polity Press.

Caïra, O. (2007). Jeux de rôle. Les forges de la fiction. Paris : CNRS Éditions.

Cesar, P. \& Geerts, D. (2011). Past, Present, and Future of Social TV: A Categorization. Dans Consumer Communications and Networking Conference (CCNC) (pp. 347351). Las Vegas : IEEE. 
Dauphragne, A. (2008). Dynamiques ludiques et logiques de genre : les univers de fantasy. Dans G. Brougère (dir.), La Ronde des jeux et des jouets. Harry, Pikachu, Superman et les autres (pp. 43-58). Paris : Autrement.

Dauphragne, A. (2011a). La fantasy au sein de la culture ludique : quand la fiction se donne à jouer. Lecture Jeune 138, 26-30. Disponible à : http://www.lecturejeunesse. org/articles/la-fantasy-au-sein-de-la-culture-ludique-quand-la-fiction-se-donne-ajouer/

Dauphragne, A. (2011b). Le sens de la fiction ludique : jeu, récit et effet de monde. Strence 2. Disponible à : https://journals.openedition.org/strenae/312. doi : https:// doi.org/10.4000/strenae. 312

De Canteloube, X. (2015, 12-14 juin). Le scénario de jeu de rôle : changer de paradigme et replacer les personnages au cœur de l'action [communication orale]. Journées d'études « Les Quarante ans du Jeu de Rôle », Villetaneuse, Paris, France. http:// www.univ-paris3.fr/medias/fichier/programme-je-jeux-de-roles_1433235548103. pdf

De Canteloube, X. (2019). Sortir du paradigme du scénario et replacer les personnages au cœur de l'action. Dans D. André \& A. Quadrat (dir.), Le Jeu de rôle sur table, un laboratoire de l'imaginaire (pp. 167-178). Paris : Lettres modernes Minard.

Dumazedier, J. (1974). Sociologie empirique du loisir. Paris : Seuil.

Dumouchel, R. (1991). Le Spectacteur et le contactile. Cinémas 1(3), 38-60. doi : https://doi.org/10.7202/1001065ar

Fine, G. A. (2002 [1981]). Shared Fantasy. Role-Playing Games as Social World. Chicago/Londres : University of Chicago Press.

Granjon, F. (2012). Reconnaissance et usages d'Internet. Une sociologie critique des pratiques de l'informatique connectée. Paris : Presses des Mines.

Huizinga, J. (1951 [1938]). Homo ludens. Essai sur la fonction sociale du jeu. Paris : Gallimard.

Rebillard, F. (2007). Le Web 2.0 en perspective, une analyse socio-économique de l'internet. Paris : L'Harmattan.

Roux, U. (2016). Jeu de rôle de table 2.0 : évolution d'une pratique ludique à l'ère du numérique. Sciences du jeu 6. Disponible à : https://journals.openedition.org/ $\operatorname{sdj} / 741$. doi : https://doi.org/10.4000/sdj.741

Roux, U. (sous presse). Jeu de rôle sur table : proposition d'une modélisation des phases de création du personnage de fiction ludique. Dans D. André \& A. Quadrat (dir.), Jeu de Rôle : Engagements et Résistances.

Roux, U. \& Roques, N. (2018). Jeu de rôle sur table 2.0 : du salon aux plateformes de streaming. Sciences du jeu 10. Disponible à : https://journals.openedition.org/ sdj/1449. doi : https://doi.org/10.4000/sdj.1449

Roux, U., Roques, N. \& Savard, S. (2018). Tabletop Role-playing Game and Technology: How Streaming has Extended the Participation Frames and Presence Planes of the Game. Going Live, Montréal (Concordia University).

Salen, K. \& Zimmerman, E. (2004). Rules of Play. Game Design Fundamentals. Cambridge : MIT Press.

Schmitt, L. (2015). Le « transmédia », un « label » promotionnel des industries culturelles toujours en cours d'expérimentation. Les Enjeux de l'information et de la communication 2(16), 5-17. doi : https://doi.org/10.3917/enic.018.0005 
Shulga, T. (2003). Présence médiatisée et construction de l'espace d'interaction : Comparaison entre jeux de rôles classiques et MMORPG. Les Cahiers du numérique $4(2), 101-115$.

Sjöblom, M., \& Hamari, J. (2017). Why Do People Watch Others Play Video Games? An Empirical Study on the Motivations of Twitch Users. Computers in Human Behaviors 75, 985-996. doi : https://doi.org/10.1016/j.chb.2016.10.019

Sjöblom, M., Törhönen, M., Hamari, J., \& Macey, J. (2017). Content Structure is King: An Empirical Study on Gratifications, Game Genres and Content Type on Twitch. Computers in Human Behavior 73, 161-171. doi : https://doi.org/10.1016/j. chb.2017.03.036

Sjöblom, M., Törhönen, M., Hamari, J., \& Macey, J. (2019). The Ingredients of Twitch Streaming: Affordances of Game Streams. Computers in Human Behavior 92, 2028. doi : https://doi.org/10.1016/j.chb.2018.10.012

Tychsen, A., Hitchens, M., Brolund, T. \& Kavakli, M. (2005). The Game Master. Dans Y. Pisan (dir.), IE '05: Proceedings of the Second Australasian Conference on Interactive Entertainment (pp. 215-222). Sydney: Creativity \& Cognition Studios Press.

Voelckel, A.-C. (1995). Jouer ensemble. Approche biographique d'un loisir : le jeu de rôle. Sociétés Contemporaines 1(21), 57-74. doi : https://doi.org/10.3406/socco.1995.1419

Weissberg, J.-L. (1999). Présences à distance. Déplacement virtuel et réseaux numériques : pourquoi nous ne croyons plus à la télévision. Paris : L'Harmattan.

\section{(9) $(1) \Theta$} «Attibution - pas d'utilisation Commerciale - Pas de Modification 4.0. International» (CC BY-NC-ND) 